\title{
Experimental determination of electron and hole mean drift distance: Application to chemical vapor deposition diamond
}

\author{
Marco Marinelli, E. Milani, G. Pucella, A. Tucciarone, and G. Verona-Rinatia) \\ INFM-Dipartimento di Ingegneria Meccanica, Università di Roma "Tor Vergata," Via del Politecnico 1, \\ I-00133 Roma, Italy \\ M. Angelone and M. Pillon \\ Associazione EURATOM-ENEA sulla Fusione, Via E. Fermi 27, I-00044 Frascati-(Roma), Italy
}

(Received 19 February 2003; accepted 23 April 2003)

\begin{abstract}
A simple technique for measuring the electron and hole mean drift distance in chemical vapor deposition polycrystalline diamond in the as-grown and in the so-called pumped state obtained by ${ }^{90} \mathrm{Sr} \beta$-particle irradiation is presented. To this purpose, the efficiency $\eta$ of a diamond-based particle detector was measured using a 5.5-MeV ${ }^{241} \mathrm{Am} \alpha$-particle source. In particular, two different experimental setups were specifically designed and realized in order to perform a systematic study of the device efficiency as a function of the $\alpha$-particle penetration depth, both in the positive and negative bias polarization. In the first setup, air is used as an absorbing layer in order to change the energy of the impinging $\alpha$ particles, while in the second one, the measurements were performed in vacuum and the incidence angle was varied in the $0^{\circ}-80^{\circ}$ range. The advantages of the latter setup are evidenced. The theoretical formula for the mean drift distances of carriers is derived using a properly modified Hecht model, and fitted to the data, allowing a separate evaluation of the charge collection distances of each carrier type $\left(\lambda_{e}=\mu_{e} \tau_{e} \mathrm{E}\right.$ and $\left.\lambda_{h}=\mu_{h} \tau_{h} \mathrm{E}\right)$. The obtained results unambiguously show that the pumping process is much more effective on hole conduction, $\lambda_{h}$ being much greater than $\lambda_{e}$ in the pumped state. (C) 2003 American Institute of Physics.
\end{abstract}

[DOI: $10.1063 / 1.1586475$ ]

Chemical vapor deposition (CVD) diamond has been shown to be a suitable material for a great number of technological applications, such as particle and UV radiation detectors, ${ }^{1-4}$ and even more so if it is driven in the so-called pumped state by ionizing irradiation, which is known to drastically improve the transport properties of synthetic diamond. ${ }^{5,6}$ However, despite the large diffusion of the procedure, few authors have carefully studied the physical grounds of the pumping process in order to discriminate its effect on electron and hole propagation in the detection process, ${ }^{7-12}$ so that very little is known to date about the contribution of each carrier type to the transport mechanism in the as-grown and pumped states.

The aim of the present work is to carefully separate and evaluate the charge collection distance of electrons and holes in polycrystalline CVD diamond, both in the as-grown and pumped states, thus giving a better insight on the transport properties of such material. To this purpose, a 130- $\mu \mathrm{m}$-thick film was grown in our labs by microwave plasma chemical vapor deposition and a particle detector was realized and tested with a ${ }^{241} \mathrm{Am} \quad \alpha$-particle source, as described elsewhere. ${ }^{13}$ The mean drift distance of each carrier type can be separately obtained by systematically studying the efficiency $\eta$ of the device as a function of the incident particle penetration depth $G$, and by analyzing the experimental curves in the framework of a properly modified Hecht model. Indeed, the contribution of one type of carrier (de-

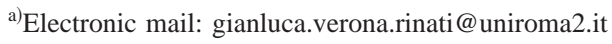

pending on bias polarity) gradually decreases as the penetration depth is reduced.

A crucial point of this work was the design and realization of a suitable experimental procedure in order to carefully control the $\alpha$-particle penetration depth in the CVD diamond. For this purpose, two different approaches are proposed and discussed. In a first experiment, an absorbing layer with increasing thickness is used in order to progressively reduce the energy of the impinging $\alpha$ particle. This was done by simply measuring the $\alpha$ spectra in air, at different sourceto-sample distances. The residual energy released in the sample by the $\alpha$ particles, and hence the values of the penetration depth $G$, were estimated using a standard nuclear physics simulation program (SRIM). In the second experimental setup, the sample is mounted inside a vacuum chamber on a rotating holder in such a way that the incidence angle $\theta$ of the $\alpha$-particle can be varied in a $0^{\circ}-90^{\circ}$ range with respect to the sample surface. Of course, in this case, $G$ is reduced by a factor $\cos \theta$ with respect to the normal incidence value $G_{0}=13.54 \mu \mathrm{m}$.

The efficiency of the diamond-based device is then measured using these techniques both in the as-grown and pumped states and under positive and negative bias polarization. All the measurements were done using a $1-\mathrm{V} / \mu \mathrm{m}$ applied electric field. Typical $\alpha$ spectra measured on our samples are reported in Refs. 7 and 13. The obtained results are summarized in Figs. 1 and 2 for air and tilt measurements, respectively. The highest penetration depth value in Fig. 1 is obtained by measuring the sample efficiency in vacuum, both in the as-grown and pumped states.

It is evident that very similar behavior of the efficiency 


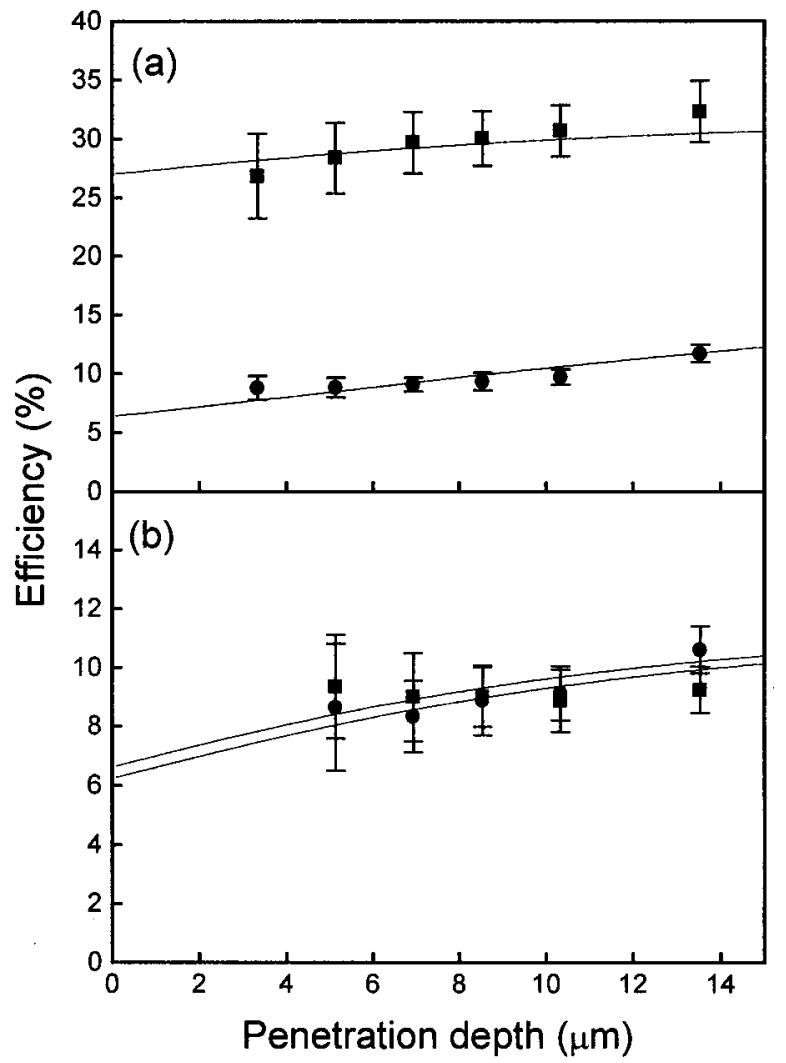

FIG. 1. Efficiency curves as a function of the penetration depth $G$ for air measurements in positive (square) and negative (circle) bias polarity; (a) in the pumped state and (b) in the as-grown state. Solid lines are the fit curves.

curves was observed in both cases. Nevertheless, as far as Fig. 1 is concerned, the higher the sample-to- $\alpha$-particle source distance, the lower the energy released in the sample. As a consequence, the measured spectrum is shifted to the lower-energy side and the signal-to-noise ratio decreases significantly. This effect is evident in Fig. 1, where the error bars become increasingly higher at low penetration depth, making measurements eventually impossible at low values of $G$, especially in the as-grown state. In the tilt measurements (Fig. 2), the total energy released by the impinging particles stays constant for all the $\theta$ values, so that both the shape and the peak position of the $\alpha$ spectra are not greatly affected by the tilting procedure. This is why, in this case, lower penetration depth regions can be reached with approximately constant error bars on the efficiency measurements.

Let us discuss now the obtained results from the transport mechanism point of view. For this purpose, a modified Hecht model is derived for a homogeneous parallel plate detector with blocking contacts. We assume that the detector is positively biased when a positive voltage is applied to the growth surface (top electrode). The mean drift distance $l_{e}(x)$ of an electron generated at a distance $x$ from the top electrode of a positively biased detector is

$$
l_{e}(x)=\frac{\int_{0}^{x} s P(s) d s+x \int_{x}^{\infty} P(s) d s}{\int_{0}^{\infty} P(s) d s},
$$

where $P(s)=e^{-s / \lambda_{e}}$ is the probability for an electron to drift for a distance $s$ [which cannot exceed the distance $x$ from the electrode, hence Eq. (1)] in an infinite material. $\lambda_{e}=\mu_{e} \tau_{e} E$

is the charge collection distance associated with the electron, $\left.\left.\quad+\lambda_{h}^{2} e^{-D / \lambda_{h}}\left(e^{G / \lambda_{h}}-1\right)\right]\right\}$
Downloaded 22 Jul 2003 to 160.80 .91 .68 . Redistribution subject to AIP license or copyright, see http: $\}$ ojps.aip.org/aplo/aplcr.jsp

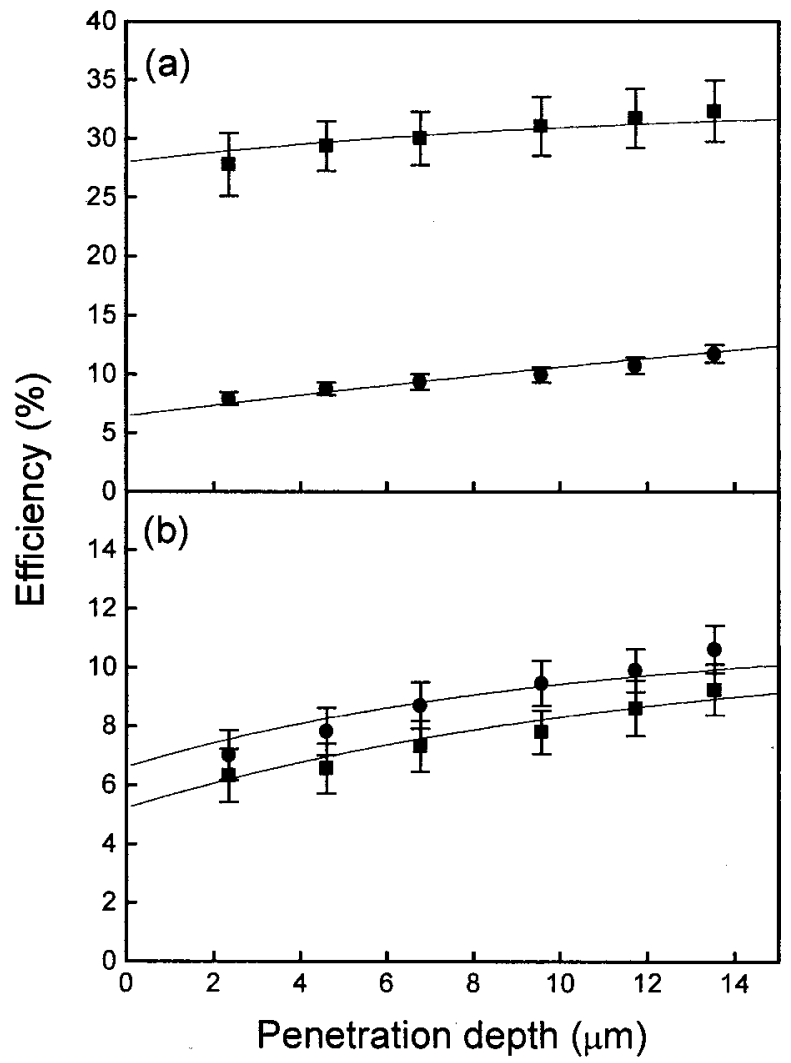

FIG. 2. Efficiency curves as a function of the penetration depth $G$ for tilt measurements in positive (square) and negative (circle) bias polarity; (a) in the pumped state and (b) in the as-grown state. Solid lines are the fit curves.

where $\mu_{e}$ is the electron mobility, $\tau_{e}$ is the electron lifetime, and $E$ the electric field. A similar formula holds for holes. The calculated results for electrons and holes drifting in a positively biased detector whose thickness is $D$ are

$$
l_{e}(x)=\lambda_{e}\left(1-e^{-x / \lambda_{e}}\right) ; l_{h}(x)=\lambda_{h}\left(1-e^{(D-x) / \lambda_{h}}\right) .
$$

The average drift distances over the whole detector are calculated by averaging over all the generated electron-hole pairs, using the energy density $u(x)$ associated with the impinging particles: $L_{e}=\left\langle l_{e}\right\rangle_{u(x)}$ and $L_{h}=\left\langle l_{h}\right\rangle_{u(x)} . L_{e}$ and $L_{h}$ are not directly experimentally accessible, but are correlated to the efficiency $\eta$ of the detector by

$$
\eta=\frac{L_{e}+L_{h}}{D} .
$$

Since the Bragg energy distribution $u(x)$ associated with an $\alpha$ particle is approximated well by the exponential function $u(x)=A+B \cdot e^{x / C}$ with a cutoff at the penetration depth $G$, the efficiency $\eta$ for positive polarization is as follows:

$$
\begin{aligned}
\eta= & \frac{\lambda_{e}+\lambda_{h}}{D}-\frac{1}{D\left[A G+B C\left(e^{G / C}-1\right)\right]} \\
& \cdot\left\{B C \left[\lambda_{e} \frac{e^{\left(1 / C-1 / \lambda_{e}\right) G}-1}{1-C / \lambda_{e}}\right.\right. \\
& \left.+\lambda_{h} e^{-D / \lambda_{h}} \frac{e^{\left(1 / C+1 / \lambda_{h}\right) G}-1}{1+C / \lambda_{h}}\right]+A\left[-\lambda_{e}^{2}\left(e^{\left.-G / \lambda_{e}-1\right)}\right.\right. \\
& \left.+\lambda_{h}^{2} e^{-D / \lambda_{h}}\left(e^{\left.G / \lambda_{h}-1\right)}\right]\right\}
\end{aligned}
$$


TABLE I. Transformations of the parameters $A, B, C$, and $G$, relative to translations and compressions of $u(x) . A_{0}, B_{0}, C_{0}$, and $G_{0}$ values are calculated for normal incident 5.5-MeV $\alpha$ particles.

\begin{tabular}{cccc}
\hline \hline & $\begin{array}{c}\text { Normal incidence } \\
\text { (vacuum) }\end{array}$ & $\begin{array}{c}\text { Translation } \\
\text { (air) }\end{array}$ & $\begin{array}{c}\text { Compression } \\
\text { (tilt) }\end{array}$ \\
\hline$A$ & $A_{0}=267.7 \mathrm{keV} / \mu \mathrm{m}$ & $A_{0}$ & $A_{0} G_{0} / G$ \\
$B$ & $B_{0}=11.17 \mathrm{keV} / \mu \mathrm{m}$ & $B_{0} e^{\left(G_{0}-G\right) / C_{0}}$ & $B_{0} G_{0} / G$ \\
$C$ & $C_{0}=3.45 \mu \mathrm{m}$ & $C_{0}$ & $C_{0} G / G_{0}$ \\
$G$ & $G_{0}=13.54 \mu \mathrm{m}$ & $G$ & $G$ \\
\hline \hline
\end{tabular}

Obviously, in the case of a negatively polarized detector, the roles of electrons and holes are interchanged. Equation (4) reduces to the usual Hecht formula when $u(x)$ is constant $(B=0$ and $G=D)$ and $\lambda_{e}=\lambda_{h}$.

In order to analyze the efficiency curves reported in Figs. 1 and 2, a few additional considerations have to be made. The energy distribution $u(x)$ is strongly dependent on the geometry of the adopted experimental setup. In the standard case (normal incidence in vacuum) the parameters $A=A_{0}$, $B=B_{0}, C=C_{0}$, and $G=G_{0}$ were calculated by fitting the SRIM simulated Bragg energy distribution with the function $u(x)$ (see Table I). On the other hand, when the $\alpha$-particle penetration depth is changed either by an air absorbing layer or by a tilting procedure, the function $u(x)$ must be properly modified. In the former case, a cutoff is to be introduced in the low-energy side of $u(x)$ corresponding to an axis shift of $G_{0}-G$, where $G$ is the new penetration depth. In the latter case, $G=G_{0} \cos \theta$, so that the $x$ axis is compressed by a factor $\cos \theta$. The corresponding transformations of the geometrical parameters $A, B, C$, and $G$ are also summarized in Table I.

The experimental curves reported in Figs. 1 and 2 were then fitted by Eq. (4) using the proper transformations of $A$, $B, C$, and $G$. Very good agreement is observed between the theoretical curves and the experimental data. It is worth noting that the curves relative to both positive and negative polarization are simultaneously fitted in each case by the very same values of $\lambda_{e}$ and $\lambda_{h}$, whose values are reported in Table II. Comparable values of the two fitting parameters are obtained from the two different setups, thus demonstrating

TABLE II. Values of $\lambda_{e}$ and $\lambda_{h}$ obtained by the fitting procedure for both experimental setups.

\begin{tabular}{lrrrrr}
\hline \hline & \multicolumn{2}{c}{ Air } & & \multicolumn{2}{c}{ Tilt } \\
\cline { 2 - 3 } \cline { 5 - 6 } \cline { 5 - 6 } & As-grown & Pumped & & As-grown & Pumped \\
\hline$\lambda_{e}[\mu \mathrm{m}]$ & $8.6 \pm 1.0$ & $8.3 \pm 1.3$ & & $8.6 \pm 0.6$ & $8.4 \pm 1.0$ \\
$\lambda_{h}[\mu \mathrm{m}]$ & $8.1 \pm 1.0$ & $36.1 \pm 1.5$ & & $6.8 \pm 0.6$ & $37.6 \pm 1.1$ \\
\hline \hline
\end{tabular}

their experimental reliability. These charge collection distances are intrinsic properties of the material used and describe well the contribution to the transport mechanism due to each carrier type. From our data, it is evident that the pumping process is confirmed to be much more effective on hole conduction, $\lambda_{h}$ being much greater than $\lambda_{e}$ in the pumped state. ${ }^{7}$

In conclusion, electron and hole mean drift distance in CVD polycrystalline diamond were carefully studied in the as-grown and the so-called pumped state. Two different experimental setups were specifically designed and realized. The tilt method was more accurate than the air measurement, and allowed the device characterization in a wider range of penetration depths. Electron and hole contributions to the conduction mechanism were successfully separated and evaluated by fitting the efficiency curves, obtained in both device polarizations, in the as-grown and pumped states, with a properly modified Hecht model. As a result, the pumping process is confirmed to be much more effective on hole conduction, $\lambda_{h}$ being much greater than $\lambda_{e}$ in the pumped state. Work is in progress to test such experimental determinations of the physical transport properties of CVD diamond with much more penetrating ionization sources, such as high energy ions.

${ }^{1}$ L. S. Pan, S. Han, D. R. Kania, S. Zhao, K. K. Gan, H. Kagan, R. Kass, R. Malchow, F. Morrow, W. F. Palmer, C. Wite, S. K. Kim, F. Sannes, S. Schnetzer, R. Stone, G. B. Thomson, Y. Sugimoto, A. Fry, S. Kanda, S. Olsen, M. Franklin, J. W. Ager III, and P. Pianeta, J. Appl. Phys. 74, 1086 (1993).

${ }^{2}$ F. Borchelt, W. Dulinski, K. K. Gan, S. Han, J. Hassard, A. Howard, H. Kagan, D. R. Kania, R. Kass, G. Lu, E. Nygard, L. S. Pan, S. Schnetzer, R. Stone, J. Straver, R. J. Tesarek, W. Trischuk, P. Weilhammer, C. White, R. L. Woodin, and S. Zhao, Nucl. Instrum. Methods Phys. Res. A 354, 318 (1995).

${ }^{3}$ P. Bergonzo, F. Foulon, R. D. Marshall, C. Jany, A. Brambilla, R. D. McKeag, and R. B. Jackman, Diamond Relat. Mater. 8, 952 (1999).

${ }^{4}$ R. D. McKeag, S. S. M. Chan, and R. B. Jackman, Appl. Phys. Lett. 67, 2117 (1995).

${ }^{5}$ T. Benke, A. Oh, A. Wagner, W. Zeuner, A. Bluhm, C. P. Klages, M. Paul, and L. Shaefer, Diamond Relat. Mater. 7, 1553 (1998).

${ }^{6}$ M. Marinelli, E. Milani, A. Paoletti, A. Tucciarone, G. Verona Rinati, M. Angelone, and M. Pillon, Appl. Phys. Lett. 75, 3216 (1999).

${ }^{7}$ M. Marinelli, E. Milani, A. Paoletti, A. Tucciarone, G. Verona Rinati, M. Angelone, and M. Pillon, Phys. Rev. B 64, 195205 (2001).

${ }^{8}$ C. Manfredotti, F. Fizzotti, A. LoGiudice, P. Polesello, E. Vittone, R. Lu, and M. Jaksic, Diamond Relat. Mater. 8, 1597 (1999).

${ }^{9}$ R. Lu, C. Manfredotti, F. Fizzotti, E. Vittone, and M. Jaksic, Mater. Res. Bull. 36, 47 (2001).

${ }^{10}$ C. Manfredotti, F. Fizzotti, A. Lo Giudice, C. Paolini, and E. Vittone, R. Lu. Nucl. Instrum. Methods Phys. Res. B 187, 566 (2002).

${ }^{11}$ C. Manfredotti, E. Vittone, F. Fizzotti, A. Lo Giudice, and C. Paolini, Diamond Relat. Mater. 11, 446 (2002).

${ }^{12}$ M. Bruzzi, D. Menichelli, S. Sciortino, and L. Lombardi, J. Appl. Phys. 91, 5765 (2002).

${ }^{13}$ M. Marinelli, E. Milani, A. Paoletti, A. Tucciarone, G. Verona-Rinati, M. Angelone, and M. Pillon, J. Appl. Phys. 89, 1430 (2001). 